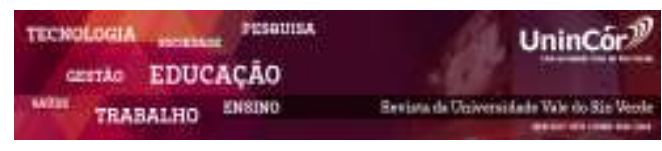

Revista da Universidade Vale do Rio Verde ISSN: 1517-0276 / EISSN: 2236-5362 Vol. 16 | n. 2 | Ano 2018

Amanda Maria Chaves Albuquerque Universidade Estadual Vale do Acaraú (UVA) amanda_albuquerque12@hotmail.com

Jailson Brito Lopes Moreira Santa Casa de Misericórdia de Sobral jailsonlopesmoreira@hotmail.com

Maria Lúcia Ribeiro de Farias Secretaria da Saúde de Reriutaba, Ceará lucia-farias28@hotmail.com

Naiana Feitosa Nogueira

Secretaria da Saúde de Reriutaba, Ceará naianafeitosa@hotmail.com

Antonia Eliane de Araújo

Secretaria da Saúde de Reriutaba, Ceará aeasousa@hotmail.com

Lucineide Paulo de Carvalho

Secretaria da Saúde de Reriutaba, Ceará neide_lpc@hotmail.com

Vitória Ferreira do Amaral Universidade Estadual Vale do Acaraú (UVA) vyctoriaamaral@gmail.com

Francisco Rosemiro Guimarães Ximenes Neto Universidade Estadual Vale do Acaraú (UVA) rosemironeto@gmail.com

\section{ANÁLISE EPIDEMIOLÓGICA DA HANSENÍASE NO MUNICÍPIO DE RERIUTABA - CEARÁ, 2001 A 2016}

\begin{abstract}
RESUMO
O presente estudo objetiva realizar análise epidemiológica e operacional da hanseníase a partir da base de dados municipal. Alicerçado em uma pesquisa descritiva, de série temporal, retrospectiva e de base documental, com casos notificados no período de primeiro de janeiro de 2001 a 31 de dezembro de 2016, na base de dados do Sistema de Informação de Agravos de Notificação (SINAN), da Secretaria Municipal da Saúde de Reriutaba, obtendo 118 casos notificados e residentes no município. Os casos novos de hanseníase da série histórica apontam o seguinte: $74,6 \%$ são multibacilares; $100 \%$ (118) dos contatos foram examinados; $58,4 \%$ (69) são do sexo masculino; 2,5\% (três) são de menores de 15 anos; 35,6\% (42) de adultos jovens e medianos, em plena idade produtiva, e 28,8\% (34) em idosos com 65 e mais anos. O coeficiente de detecção varia de média $(0,47)$ para hiperendemicidade $(8,95)$. Os resultados evidenciaram a necessidade do município de reavaliar as ações que vêm sendo realizadas e planejar novas estratégias, com o objetivo de corrigir os erros e reorganizar as ações, para que o processo de combate à doença seja agilizado.
\end{abstract}

Palavras-chave: Hanseníase. Epidemiologia. Doenças Endêmicas.

\section{EPIDEMIOLOGICAL ANALYSIS OF LEPROSY IN THE MUNICIPALITY OF RERIUTABA - CEARÁ, 2001 TO 2016}

\begin{abstract}
The present study aims to perform epidemiological and operational analysis of leprosy from the municipal database. Based on a descriptive, retrospective, documental-based, retrospective and case-control study, with cases reported from January 1, 2001 to December 31, 2016, in the SINAN database, of the Municipal Health Department of Reriutaba, obtaining 118 cases notified and resident in the municipality. New cases of leprosy in the historical series indicate the following: $74.6 \%$ are multibacillary; $100 \%$ (118) of the contacts were examined; $58.4 \%$ (69) are males; $2.5 \%$ (three) are under 15 years of age; $35.6 \%$ (42) of young and middle-aged adults, at full productive age, and $28.8 \%$ (34) in the elderly aged 65 and over. The detection coefficient varies from everage (0.47) to hyperendemicity (8.95). The results evidenced the need of the municipality to reassess the actions that have been carried out and to plan new strategies, with the objective of correcting the errors and reorganizing the actions, so that the process of combating the disease becomes faster.
\end{abstract}

Keywords: Leprosy. Epidemiology. Endemic Diseases. 


\section{INTRODUÇÂO}

A hanseníase é caracterizada como uma doença de curso crônico, infectocontagiosa, causada pelo bacilo Mycobacterium leprae, que tem potencial de alta infectividade, porém baixa patogenicidade, o que torna capaz de infectar vários sujeitos e por isso, poucos desenvolvem a doença. A transmissão do bacilo da hanseníase ocorre a partir de um sujeito infectado e sem tratamento, por meio da eliminação do bacilo pelas vias aéreas superiores (mucosa nasal e orofaringe), para outro sujeito que mantém contato próximo e prolongado (BRASIL, 2016a).

A hanseníase é considerada uma doença de diagnóstico fácil na maioria das vezes, principalmente, quando as clássicas lesões de pele estão presentes. Contudo, o diagnóstico dos sujeitos que não apresentam lesões cutâneas, conhecidas como formas neurais puras, "representam um amplo desafio, [...] que demanda ampla e demorada investigação, uma vez que não há nenhum exame laboratorial confirmatório rápido, fácil e universalmente acessível” (PINHEIRO, 2014, p. 10).

Por conta de sua amplitude e magnitude, a hanseníase faz parte do rol da Lista Nacional de Doenças de Notificação Compulsória, portanto, os casos diagnosticados devem ser obrigatoriamente notificados e investigados, de acordo com a Portaria No 204 e Portaria No 205, de fevereiro de 2016, do Ministério da Saúde (BRASIL, 2016b).

Alguns dos maiores desafios enfrentados frente à erradicação e eliminação da hanseníase está relacionado à escassez de novas pesquisas, quanto à prevenção, tratamento e diagnóstico precoce da doença, pois as recomendações mundiais permanecem baseadas em estratégias formuladas há décadas. O tratamento com a poliquimioterapia (PQT), que é o indicado para os sujeitos diagnosticados com hanseníase, é o mesmo desde o início dos anos 1980 (CRUZ et al., 2017). Outro importante desafio para eliminação da doença, é a inexistência de tratamento, com medicação mais eficaz em menor tempo.

Contudo, algumas ações têm contribuído para a redução e controle da carga da hanseníase no Brasil. Apesar de não existir uma proteção específica para a hanseníase, algumas ações podem ser desenvolvidas para ajudar no controle desta, como a educação em saúde com a população, sobre as formas de prevenção, controle e detecção precoce. Além de uma efetiva investigação epidemiológica, procurando diagnosticar de forma precoce os casos novos; realização do tratamento adequado, realizando-o até a alta por cura; prevenção e tratamento de incapacidades; e a realização de exame nos contatos, orientações e aplicação da vacina BCG (Bacillus Calmette-Guérin) são estratégias necessárias e eficientes no controle da hanseníase (BRASIL, 2016a; BRASIL, 2016b).

Nas últimas três décadas vêm se alcançando um maior controle da hanseníase em países considerados endêmicos, mas apesar disso a hanseníase ainda é considerada um problema de saúde pública, inclusive no Brasil, pois a detecção de casos novos é contínua, o que 
dificulta o alcance da meta estabelecida pela Organização Mundial da Saúde (OMS), que é a prevalência inferior a um caso a cada dez mil habitantes. O Brasil está entre os três países em nível mundial que notificam mais de 10.000 novos casos de hanseníase anualmente. No ano de 2016 foram notificados 25.218 casos novos no Brasil, com uma taxa de detecção de 12,2/100mil habitantes, enquadrando em parâmetros hiperendêmico (BRASIL, 2016a; BRASIL, 2018; OMS, 2016).

A região Nordeste do Brasil está entre as três regiões com maior coeficiente de detecção de casos novos de hanseníase. Entre o período de 2003 a 2012 obteve-se o percentual de 25,8 casos novos por 100 mil habitantes, apresentando uma alta endemicidade. O Estado do Ceará, que se localiza nessa região, também se destaca devido ao grande número de casos novos notificados, somente no ano de 2013 foram notificados 2.069 casos novos, resultando em um coeficiente de detecção de 24 casos novos por 100 mil habitantes (CEARÁ, 2014).

Os indicadores epidemiológicos da hanseníase no município de Reriutaba, no estado do Ceará, apontam para uma situação endêmica e, que necessita de ações voltadas para o controle e eliminação da doença. Assim, o presente estudo objetiva realizar uma análise epidemiológica e operacional da hanseníase, a partir da base de dados municipal.

\section{MATERIAL E MÉTODOS}

Estudo descritivo, de série temporal, retrospectivo e de base documental, sob abordagem quantitativa, realizado com base nos registros de casos de hanseníase de Reriutaba Ceará, entre janeiro e junho de 2017, com os 118 casos notificados no SINAN, durante o período de $1^{\circ}$ de janeiro de 2001 a 31 de dezembro de 2016, residentes no município.

Reriutaba é um do Estado do Ceará, localizado na Mesorregião do Noroeste Cearense, na Microrregião de Ipu. Em 2010, o município contava com uma população estimada em 19.455 habitantes; com um Índice de Desenvolvimento Humano (IDH) de 0,601 e uma área territorial de $383.319 \mathrm{~km}^{2}$ e densidade demográfica de 50,75 (hab./ $\left.\mathrm{km}^{2}\right)$, estando localizada a aproximadamente $300 \mathrm{~km} \mathrm{de}$ distância da capital, Fortaleza - CE (IBGE, 2016).

Levando em conta como critério de inclusão apenas os casos novos/ano de residentes do município, ou seja, os sujeitos que nunca receberam qualquer tratamento específico para a hanseníase e o critério de exclusão foram os registros de casos duplicados. Os dados coletados foram sistematizados e apresentados em forma tabular, com o cálculo e avaliação de alguns indicadores para o monitoramento da hanseníase, além da avaliação epidemiológica e operacional. Os indicadores utilizados, estão descritos no Quadro 1. A discussão dos resultados foi efetuada com base na literatura pertinente à luz do referencial teórico acerca da análise epidemiológica da hanseníase.

Para realização do estudo, foram observados os aspectos éticos e legais da pesquisa de acordo com a Resolução de $\mathrm{N}^{\circ}$ 466/2012 do Conselho Nacional de Saúde (CNS), mas por se tratar de um estudo apenas com dados secundários, de livre acesso e 
domínio público, sem identificação dos

e aprovação pelo Comitê de Ética em Pesquisa participantes, não se fez necessário a submissão (CEP).

Quadro 1 Indicadores epidemiológicos e operacionais, com seus respectivos cálculos, utilidade e parâmetros.

\begin{tabular}{|c|c|c|c|}
\hline $\begin{array}{l}\text { Indieadores } \\
\text { Epidemiológicos }\end{array}$ & Construgaio (*) & Utilidade & Paraimetros \\
\hline $\begin{array}{l}\text { 1. Coeficiente de } \\
\text { deteçso anual } \\
\text { de casos } \\
\text { novos, por } \\
10.000 \text { hab. }\end{array}$ & $\begin{array}{l}\text { Cascos novos residentes } \\
\frac{\text { diagnosticados no ano }}{\text { Populasto total }} \text { rexulente em 01/07/ano } \\
\text { rexide }\end{array}$ & $\begin{array}{l}\text { Determinar a } \\
\text { tendéncia secular } \\
\text { da endemia e medir } \\
\text { a intensidade das } \\
\text { atividades de } \\
\text { derogaso dos casos }\end{array}$ & $\begin{array}{l}\text { Hiperentémico } \geq 4,0 / 10.000 \text { hab. } \\
\text { Muito Alro } 4,0+1,0 / 10.000 \text { hab. } \\
\text { Alto } 2,0+1,0 / 10.000 \text { hab, } \\
\text { Méctio } 1,0 \longrightarrow 0,2 / 10.000 \text { hab } \\
\text { Baiko }<0,2 / 10.000 \text { hab. }\end{array}$ \\
\hline $\begin{array}{l}\text { 2. Cocficiente de } \\
\text { deteç̧o anual } \\
\text { de casos nowos } \\
\text { na populasto de } \\
0 \text { a } 14 \text { anos, por } \\
10.000 \text { hab. }\end{array}$ & $\begin{array}{l}\text { Casos novos residentes } \\
\text { com } 0 \text { a } 14 \text { anos de idade } \\
\text { diagnosticados no ano } \\
\text { Populaça residente } \\
\text { caun idade entre } 0 \text { e } \\
14 \text { anox em 01/07/ano }\end{array}$ & $\begin{array}{l}\text { Determinar a } \\
\text { rendéncia secular } \\
\text { da endemia }\end{array}$ & $\begin{array}{l}\text { Hiperendèmico } \geq 1,0 / 10.000 \text { hab. } \\
\text { Muito Alto } 1,0 \longrightarrow 0,5 / 10.000 \text { habs. } \\
\text { Alto } 0,5+0,25 / 10.000 \text { hab. } \\
\text { Médio } 0,25 \longrightarrow 0,05 / 10.000 \text { hat } \\
\text { Haiso }<0,05 / 10.000 \text { hab. }\end{array}$ \\
\hline $\begin{array}{l}\text { Indieador } \\
\text { Operacional }\end{array}$ & Construção (*) & Utillidade & Parämetros \\
\hline $\begin{array}{l}\text { 1. Proporgáa de } \\
\text { examinadon } \\
\text { entre os } \\
\text { contatos } \\
\text { intradomiciliares } \\
\text { de casos novos } \\
\text { diagnosticados } \\
\text { no ano }\end{array}$ & $\begin{array}{l}\text { Contatos } \\
\text { intradomiciliares de } \\
\text { casas novos } \\
\text { diagnosticados no ano, } \\
\text { que foram examinados } \\
\text { Total de contatos } \\
\text { intradomicitiares de } \\
\text { casos novos } \\
\text { diagnosticados no ano }\end{array}$ & $\begin{array}{l}\text { Avaliar a execuçà } \\
\text { da arividade de } \\
\text { vigilancia de } \\
\text { contatos }\end{array}$ & $\begin{array}{l}\text { Bom } \geq 75 \% \\
\text { Regular } 50 \quad 75 \% \\
\text { Precário }>50 \%\end{array}$ \\
\hline
\end{tabular}

Fonte: BRASIL. Ministério da Saúde. Secretaria de Políticas de Saúde. Departamento de Atenção Básica. Guia para o Controle da hanseníase. Brasília: Ministério da Saúde, 2002.

*Todos os indicadores devem ser calculados utilizando-se dados de casos residentes, independentemente do local de detecção e tratamento.

Gráfico 1 Evolução da endemia da hanseníase segundo sexo e faixa etária. Reriutaba - Ceará, 2001 a 2016.

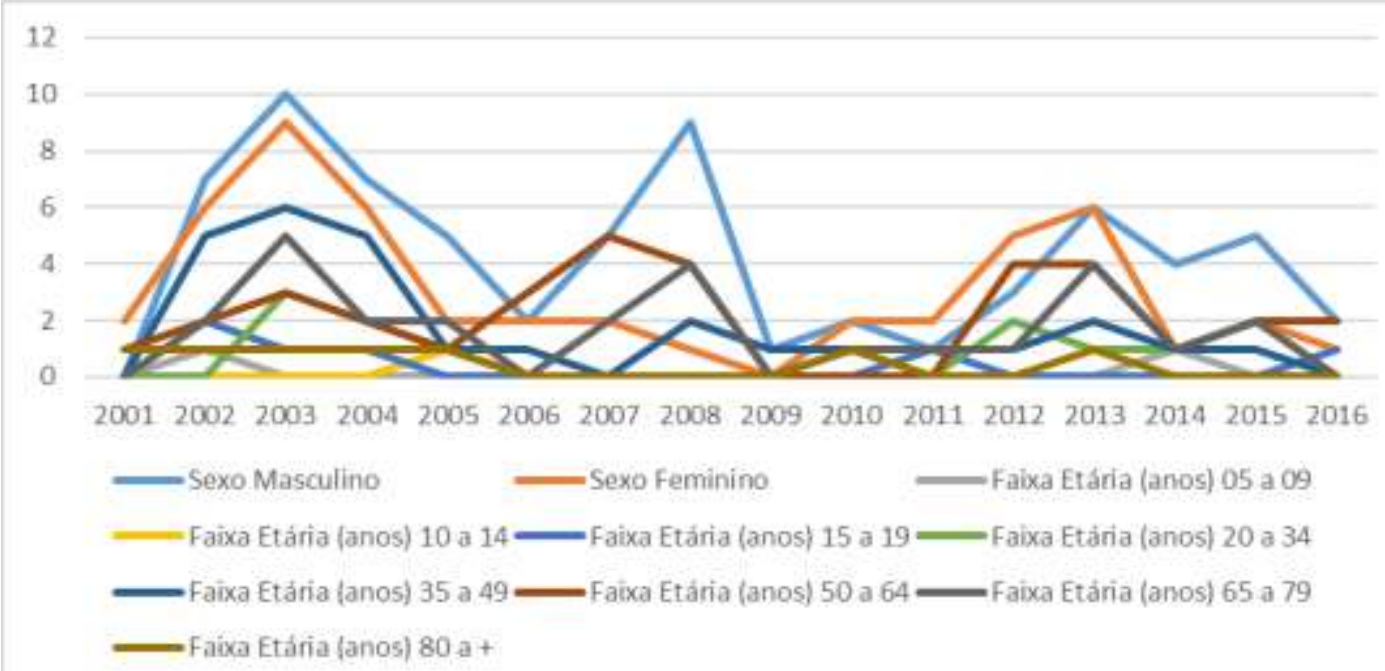

Fonte: RERIUTABA. Secretaria da Saúde. Coordenadoria de Vigilância à Saúde. Sistema de Informação de Agravos de Notificação (SINAN): Hanseníase. Reriutaba: Secretaria da Saúde, 2017. 
Gráfico 2 Evolução da endemia da hanseníase segundo a classificação operacional. Reriutaba - Ceará, 2001 a 2016.

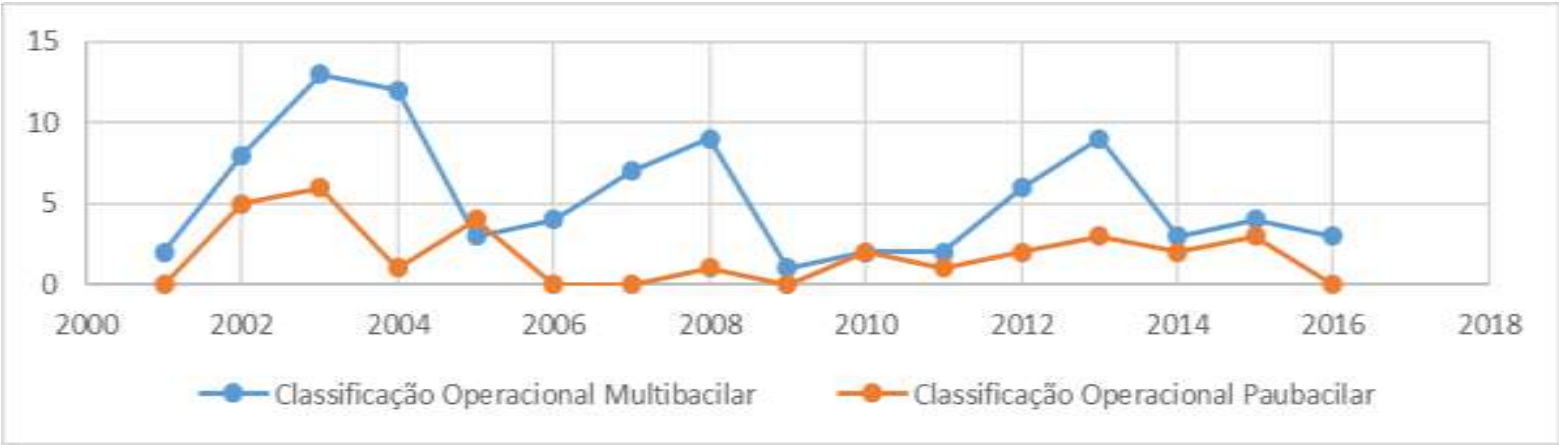

Fonte: RERIUTABA. Secretaria da Saúde. Coordenadoria de Vigilância à Saúde. Sistema de Informação de Agravos de Notificação (SINAN): Hanseníase. Reriutaba: Secretaria da Saúde, 2017.

Gráfico 3 Indicadores epidemiológicos da endemia de hanseníase. Reriutaba - Ceará, 2001 a 2016.

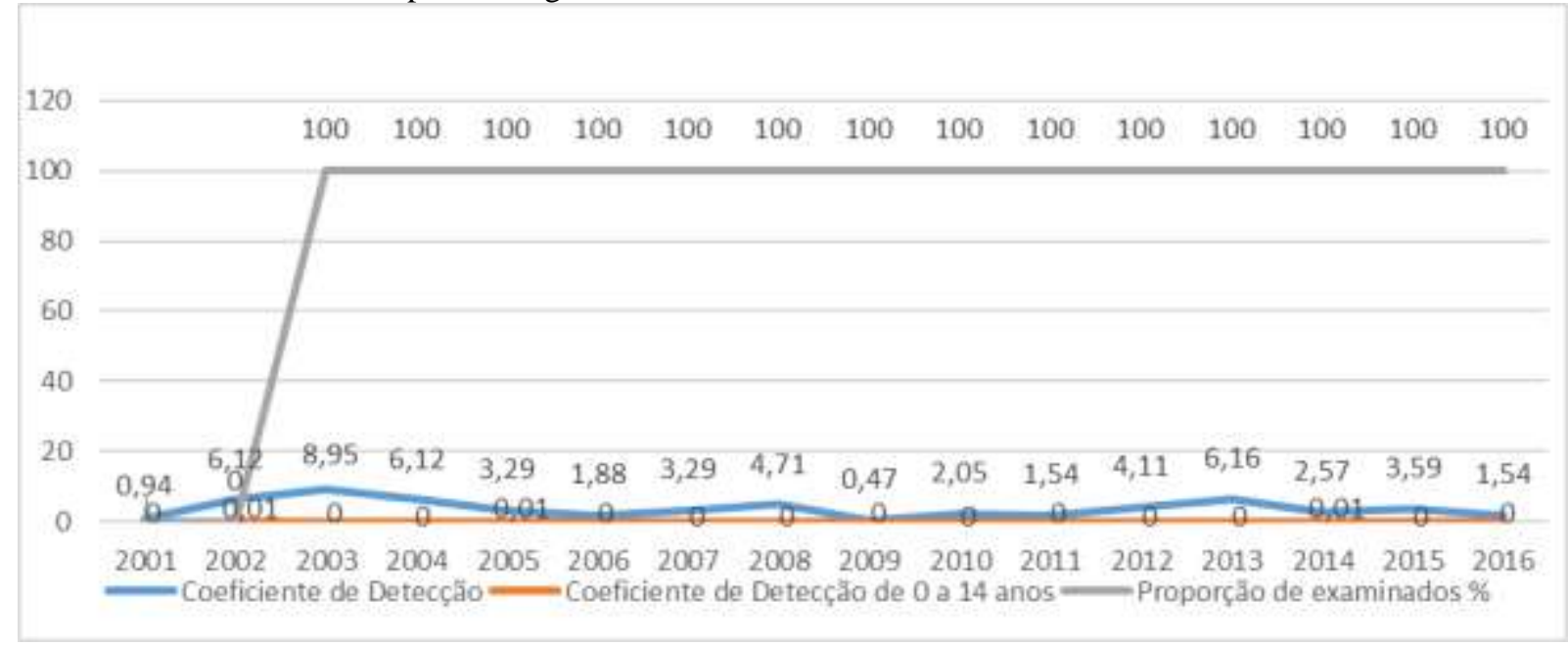

Fonte: RERIUTABA. Secretaria da Saúde. Coordenadoria de Vigilância à Saúde. Sistema de Informação de Agravos de Notificação (SINAN): Hanseníase. Reriutaba: Secretaria da Saúde, 2017.

\section{RESULTADOS E DISCUSSÃO}

O município em estudo notificou 118 casos de hanseníase, no período de 2001 a 2016, sendo $\quad 88$ multibacilares $(\mathrm{MB})$ e 30 paucibacilares $(\mathrm{PB})$, o que demonstra uma série histórica de endemicidade, como pode ser visualizado no Gráfico 1.

De acordo com o Gráfico 1, há uma predominância do sexo masculino, 58,4\% (69) dos casos novos de hanseníase. Em estudo realizado por Oliveira e Macedo (2012), no município de Iretama - Paraná, no período de 2005 a 2009, eles também detectaram uma maior predominância dos casos de hanseníase no sexo masculino, resultando em um total de $55,18 \%$ de casos novos de hanseníase, no sexo masculino, durante o período avaliado.

Os homens são clientes menos frequentes nas unidades de saúde do Sistema Único de Saúde (SUS) do que as mulheres, principalmente, na Estratégia Saúde da Família (ESF), por conta de diversos fatores que vão desde o horário de funcionamento dos Centros de Saúde coincidir com o do mercado de trabalho, até ao padrão de cuidados individual, que é mais comum às mulheres, dentre outros, o que pode acarretar nos casos de hanseníase na instalação de incapacidades permanentes, se não ocorrer um diagnóstico precoce. 
Os dados demostram que $52,5 \%$ (62) dos sujeitos diagnosticados com hanseníase ao longo dos 16 anos avaliados, estão entre a faixa etária de 35 a 64 anos, o que compõe o estrato da população economicamente ativa; além de: três (2,54\%) em menores de 15 anos; 42 (35,6\%) de adultos jovens e medianos, em plena idade produtiva, e $34(28,8 \%)$ em idosos com 65 e mais anos. Em estudo realizado por Miranzi et al. (2010), em Uberaba - Minas Gerais, no período de 2000 a 2006, aponta dados $(31,4 \%)$ semelhantes ao desse estudo, na faixa etária de 34 a 49 anos. E o somatório da faixa etária de 20 a 49 anos, foi o equivalente a $53,4 \%$. O que demonstrou que grande parte da população economicamente ativa deste município se encontra afetada pela hanseníase.

O fato da predominância de casos notificados de hanseníase acima dos 35 anos $(52,5 \%)$, assim como, a de casos multibacilares, 75\% (88), evidencia-se o longo período de incubação do Bacilo de Hansen. Segundo o Ministério da Saúde, o período de incubação da hanseníase pode durar em média de dois a sete anos, mas já foi constatado períodos mais curtos, de sete meses e mais longos, de 10 anos (BRASIL, 2016a).

Os sujeitos acometidos pela hanseníase em sua forma multibacilar, e que estão em tratamento, são os maiores responsáveis pela transmissão da doença, pois são capazes de eliminar uma grande quantidade de bacilos, cerca de 10 milhões, que ficam na mucosa nasal e são expelidos para o meio exterior na forma de aerossóis, aumentando significantemente o risco dos contatos domiciliares de contraírem a doença (BRASIL, 2016b).
Ao analisarmos o histórico dos casos de hanseníase, de acordo com a classificação operacional, é possível perceber que a ocorrência de casos multibacilares é predominante. Os casos MB equivalem a $75 \%$ (88) do total de casos notificados. O trabalho de Araújo et al. (2014), realizado em um programa de eliminação da hanseníase, no município de São Luís Maranhão, no período de 2010 a 2012, também encontrou uma maior incidência de casos multibacilares, o que demonstra possível falha na detecção precoce por parte da Rede de Atenção à Saúde (RAS), pois fica evidente que o diagnóstico, nestes casos, foram realizados tardiamente, aumentando assim, o risco de desenvolvimento de complicações neurais e incapacidades físicas nos sujeitos, aumentando a probabilidade de infectar diversos sujeitos no espaço domiciliar e comunitário.

Em estudo realizado por Ximenes Neto et al. (2013), no município de Cariré - Ceará, que faz continuidade territorial com Reriutaba, também mostrou um elevado número de casos de hanseníase multibacilar, totalizando 41,6\% (49) do total de casos analisados durante o período de 2001 a 2010. O que demonstra o alto poder de infectividade do bacilo de Hansen nessa comunidade, pois os contatos dos casos MB apresentam maior risco de contaminação, do que os contatos de casos PB.

Nesta perspectiva, fica evidente que a melhor estratégia para controle e eliminação da doença, é a busca ativa de sintomáticosdermatológicos, com a avaliação clínica, oferta da baciloscopia de Hansen e uma efetiva investigação epidemiológica, para o diagnóstico precoce dos casos, atrelado a um tratamento 
adequado, para prevenção de incapacidades e diminuição da carga de transmissão da doença e possível quebra da cadeia de transmissão comunitária. Para tanto, é imprescindível que a RAS que esteja integrada seja efetiva e resolutiva.

A série histórica em estudo mostra que $100 \%$ dos contatos foram examinados a partir de 2003 a 2016, conforme Gráfico 3. A vigilância dos contatos tem por objetivo examinar os sujeitos que tiveram contato prolongado com um caso confirmado de hanseníase (caso índice) na busca ativa de novos casos. Além de tentar descobrir a possível fonte de infecção do paciente acometido pela hanseníase, se a mesma foi do seio familiar (lar) ou do meio social (BRASIL, 2016a).

Os contatos de sujeitos com hanseníase pode ser dividido em dois tipos: o contato domiciliar e contato social. Compreende-se como contato domiciliar toda e qualquer pessoa que resida ou tenha residido com o paciente acometido pela hanseníase e não tratado, por um longo período de tempo. Já o contato social se refere às pessoas que conviva ou tenha convivido, de forma próxima e prolongada, em relações familiares ou não, com caso confirmado de hanseníase, podendo incluir vizinhos e colegas de trabalho ou escola (BRASIL, 2016b).

Neste estudo, percebe-se que o registro e exames dos contatos têm sido prioridade para as equipes da ESF, o que configura uma importante estratégia para controle e eliminação da doença. Apenas nos anos de 2001 e 2002, que não houve registro de contatos examinados, embora tenha tido casos novos notificados, o que seria muito difícil estes casos não possuírem contato com ninguém. Mas no restante dos anos houve uma quantidade expressiva de contatos registrados e todos foram examinados.

Um estudo realizado no Rio de Janeiro, no período de 1987 a 2010, concluiu que o controle dos contatos é uma importante estratégia e altamente eficaz para evoluir no combate à hanseníase e a vigilância ativa desses contatos é de suma importância em áreas com alta endemicidade, como no município de Reriutaba (HACKER et al., 2012).

O Ministério da Saúde adota, no Brasil, os seguintes parâmetros de endemicidade, baseados no coeficiente de detecção de casos por 10 mil habitantes: baixo - menor que 0,2 ; médio - 0,2 a 0,9; alto - 1,0 a 1,9; muito alto - 2,0 a 3,9; e hiperendêmico - maior ou igual a 4,0 (RIPSA, 2008). O município em estudo apresenta uma endemicidade que varia de média $(0,47)$ a hiperendemicidade $(8,95)$, o que mostra uma cadeia de transmissão efetiva, principalmente pelo elevado número de casos multibacilares.

Talhari et al. (2012) afirmam que a lenta redução da incidência da hanseníase, em países endêmicos como o Brasil, Índia e Indonésia, está estritamente relacionado às características epidemiológicas do bacilo Mycobacterium leprae, pois este possui um longo período de incubação.

Nos anos de 2007 a 2010 foram introduzidas importantes mudanças no Brasil, no que diz respeito às políticas públicas voltadas para o controle e eliminação da hanseníase. Dentre estas mudanças está a monitorização do coeficiente de detecção anual de casos novos menores de 15 anos de idade e vigilância dos contatos. Essas foram as estratégias definidas 
como mais importantes para o alcance das metas estabelecidas.

O município de Reriutaba notificou três casos (nos anos 2002, 2005 e 2014) de hanseníase em menores de 15 anos, durante o período analisado. O coeficiente de detecção de casos novos de hanseníase em menores de 15 anos é um importante indicador, pois a partir dele é possível determinar a tendência secular da endemia e medir a força recente de transmissão da doença. As unidades de saúde dos municípios têm por obrigação, diante de um caso suspeito de hanseníase em menores de 15 anos, preencher o Protocolo Complementar de Investigação Diagnóstica de Casos de Hanseníase em Menores de 15 anos (BRASIL, 2016b).

Em um estudo epidemiológico realizado no Rio de Janeiro, foram identificados grupos de risco e fatores que contribuem para o controle da hanseníase, o que proporcionou ao Ministério da Saúde no Brasil ter um embasamento científico para propor ações políticas, administrativas e investimentos em meios governamentais (TALHARI et al., 2012). Portanto, o indicador de detecção anual dos casos novos de hanseníase deve decair em decorrência das ações de controle promovidas pelos órgãos governamentais em prol da eliminação da doença, mas a diminuição deste indicador se dá de forma lenta e gradativa, pois há estratégias que auxiliam numa melhor detecção dos casos, havendo assim um aumento e sendo esse um fator de influência para o cálculo.

A tendência apresentada neste estudo, quanto ao coeficiente de detecção anual de casos novos de hanseníase, revela uma realidade preocupante frente à realidade brasileira, ao levar em consideração o não cumprimento das metas estabelecidas pelas várias estratégias e programas existentes desde o final do século passado, que foram estabelecidas com o propósito de nortear a qualidade da assistência aos sujeitos acometidos pela hanseníase.

A busca ativa é uma importante ferramenta no combate e controle da doença, por meio dela é possível identificar de forma precoce os casos novos existentes na comunidade, contribuindo com a quebra da cadeia de transmissão. Pois, é possível identificar os sujeitos com hanseníase, que abandonaram o tratamento; sendo necessário detectar a doença em sua fase inicial, evitando incapacidades, exclusão social e estigmas (BRASIL, 2016b).

Entretanto, apenas a busca ativa não soluciona o problema de endemicidade da hanseníase, ela auxilia numa melhor identificação e acompanhamento do caso. Outras ações são importantes para o controle, erradicação e eliminação da doença, tais como: educação em saúde, investigação epidemiológica, tratamento até a cura, prevenção de incapacidades, vigilância epidemiológica, registro, exame e aplicação da BCG nos contatos próximos, dentre outros.

\section{CONCLUSÕES}

A partir da análise dos indicadores é possível perceber que os coeficientes de detecção de casos novos de hanseníase estão muito altos, mesmo nos últimos anos, demonstrando que não está havendo diminuição da carga da doença no município. $\mathrm{O}$ que remete à necessidade de reavaliar as ações que vêm sendo realizadas e 
planejar novas estratégias, com o objetivo de corrigir os erros e reorganizar as ações, para que o processo de combate à doença seja agilizado.

O estudo ressaltou ainda a importância da investigação epidemiológica e vigilância dos contatos para o controle da doença e obtenção da meta estabelecida pela OMS, que é a prevalência de menos de um caso a cada dez mil habitantes.

\section{REFERÊNCIAS}

ARAÚJO, A.E.R.A.; AQUINO, D.M.C.; GOULART, I.M.B.; PEREIRA, S.R.F.P.; FIGUEIREDO, I.A; SERRA, H.O.; FONSECA, P.C.A.; CALDAS, A.J.M. Complicações neurais e incapacidades em hanseníase em capital do nordeste brasileiro com alta endemicidade. Rev Bras Epidemiol, v. 17, n. 4, p. 899 a 910, p. 2014. Citado em: 28 de maio de 2017.

Disponível em:

<http://www.scielo.br/pdf/rbepid/v17n4/pt_1415

-790X-rbepid-17-04-00899.pdf $>$.

BRASIL. Ministério da Saúde. Boletim

Epidemiológico. Brasília. Ministério da Saúde, v.49, n.4, 2018. Disponível em < http://portalarquivos2.saude.gov.br/images/pdf/2 018/janeiro/31/2018-004-Hanseniasepublicacao.pdf> . Acesso em 16 junho de 2018.

BRASIL. Ministério da Saúde. Secretaria de Vigilância em Saúde. Guia de Vigilância em Saúde. Brasília: Ministério da Saúde, 2016b.

BRASIL. Ministério da Saúde. Diretrizes para vigilância, atenção e eliminação da Hanseníase como problema de saúde pública: manual técnico-operacional. Brasília: Ministério da Saúde, 2016a.

BRASIL. Ministério da Saúde. Secretaria de Políticas de Saúde. Departamento de Atenção Básica. Guia para o Controle da hanseníase. Brasília: Ministério da Saúde, 2002. Citado em: 30 de maio de 2017. Disponível em:< http://bvsms.saude.gov.br/bvs/publicacoes/guia_ de_hanseniase.pdf $>$.

CEARÁ. Secretaria da Saúde. Coordenadoria de Promoção e Proteção à Saúde Núcleo de
Vigilância Epidemiológica. Informe

Epidemiológico Hanseníase. Ceará: Governo do Estado do Ceará, 2014. Citado em: 1 de junho de 2017. Disponível em:

<http://www.ipece.ce.gov.br/perfil_basico_muni cipal/2014/Reriutaba_Br_office.pdf $>$.

CRUZ, R. C. S. et al. Hanseníase: situação atual, aspectos clínicos e laboratoriais, história de tratamento e perspectiva da terapia multidroga uniforme para todos os pacientes. A. Bras. Dermatol Rio de Janeiro, v. 92, n. 6, p. 761-773, dez. 2017. Disponível em <http://www.scielo.br/scielo.php?script=sci_artt ext\&pid=S0365-

05962017000600761\&lng=en\&nrm=iso >. Acess o em 16 de junho de 2018.

HACKER, M.A.; DUPPRE, N.C.; NERY. J.A.C.; SARNO. E.N. Characteristics of leprosy diagnosed through the surveillance of contacts: a comparison with index cases in Rio de Janeiro, 1987-2010. Mem. Inst. Oswaldo Cruz, v. 107, n. 1, p. 49 a 54. Rio de Janeiro, 2012. Citado em: 25 de maio de 2017. Disponível em:

<http://www.scielo.br/pdf/mioc/v107s1/09.pdf>.

INSTITUTO BRASILEIRO DE GEOGRAFIA E ESTATÍSTICA (IBGE). Cidades: Reriutaba: IBGE; 2016. Citado em: 30 de maio de 2017. Disponível em:

<http://cidades.ibge.gov.br/xtras/perfil.php?lang $=\& \operatorname{codmun}=231170 \&$ search $=0 \mid$ reriutaba|infograf icos:-informacoes-completas>.

MIRANZI, S.S.C.; PEREIRA, L.H.M.; NUNES, A.A. Perfil epidemiológico da hanseníase em um município brasileiro, no período de 2000 a 2006.

Rev Soc Bras Med Trop; v. 43, p. 62 a 67, a. 2010. Citado em: 10 de maio de 2017.

Disponível em:

http://www.scielo.br/pdf/rsbmt/v43n1/a14v43n1.

OLIVEIRA, F.F.L.; MACEDO, L.C. Perfil epidemiológico dos portadores de hanseníase em um município da região centro-oeste do Paraná.

SaBios: Rev. Saúde e Biol., v. 7, n. 1, p. 45-51, 2012. Citado em: 29 de maio de 2017.

Disponível em:

file:///C:/Users/Alana/Downloads/966-5381-3PB.pdf.

OMS. Organização Mundial da Saúde. Estratégia mundial de eliminação da lepra 2016-2020:

Acelerar a ação para um mundo sem lepra.

Organização Pan-Americana da Saúde. SEARO: 
Organização Mundial da Saúde, 2016.

Disponível em:

http:<//apps.who.int/iris/bitstream/10665/208824

/8/9789290225201-Portuguese.pdf $>$. Acesso em

16 de junho de 2018 .

PEREIRA, E.V.E.; NOGUEIRA. L.T.;

MACHADO, H.A.S.; LIMA, L.A.N.; RAMOS,

C.H.M. Perfil epidemiológico da hanseníase no município de Teresina, no período de 2001-2008.

An Bras Dermatol, v. 86, n. 2, p. 235 a 240, a.

2011. Citado em: 09 de junho de 2017.

Disponível em: <

http://www.scielo.br/pdf/abd/v86n2/v86n2a05.pd

$\mathrm{f}>$.

PINHEIRO, M.G.C.; SILVA, S.Y.B.; FRANÇA, A.L.M.; MONTEIRO, B.R.; SIMPSON, C.A.

Hanseníase: uma abordagem educativa com estudantes do ensino médio. Rev. Pesqui. Cuid.

Fundam, v. 6, n. 2, p. 776 a 784, a. 2014.

Citado em: 16 de maio de 2017. Disponível em:< pesquisa.bvs.br/brasil/resource/pt/bde-25455>.

RERIUTABA. Secretaria da Saúde.

Coordenadoria de Vigilância à Saúde. Sistema de Informação de Agravos de Notificação (SINAN):

Hanseníase. Reriutaba: Secretaria da Saúde, 2017.

RIPSA. Rede Interagencial de Informação para a Saúde. Indicadores básicos para a saúde no Brasil: conceitos e aplicações. 2. ed. Brasília: Organização Pan-Americana da Saúde; 2008. Citado em: 01 de junho de 2017. Disponível em: $<$ http://tabnet.datasus.gov.br/tabdata/livroidb/2ed /indicadores.pdf $>$.

TALHARI, S.; GROSSI, M.A.F.; OLIVEIRA, M.L.W.D.R.; GONTIJO, B.; TALHARI, C.; PENNA, G.O. Hansen's disease: a vanishing disease? Mem Inst Oswaldo Cruz. v. 107 (Suppl. I): 13-16. Rio de Janeiro, 2012. Citado em: 25 de maio de 2017. Disponível em: < http://www.scielo.br/pdf/mioc/v107s1/03.pdf>.

XIMENES NETO, F.R.G.; GOMES L.B.; MARTINS F.; MARTINS A.F.; CARVALHO FILHO J.; CARLOS S.M. Epidemiologia da hanseníase no município de Cariré - Ceará 2001 a 2010. Revista Eletrônica Gestão \& Saúde, v. 4, n. 3, a. 2013, p.829-42. Citado em: 11 de junho de 2017. Disponível em: <gestaoesaude.unb.br/index.php/gestaoesaude/ar ticle/download/480/628>.
Amanda Maria Chaves Albuquerque

Graduada em Universidade Estadual Vale do Acaraú (UVA).

\section{Jailson Brito Lopes Moreira}

Residente em Enfermagem em Urgência e

Emergência da Santa Casa de Misericórdia de Sobral.

\section{Maria Lúcia Ribeiro de Farias \\ Enfermeira da Secretaria da Saúde de Reriutaba, Ceará. Especialista em Saúde da Família e Gestão Clínica.}

\section{Naiana Feitosa Nogueira \\ Enfermeira. Coordenadora da Vigilância à Saúde da Secretaria da Saúde de Reriutaba, Ceará. Especialista em Gestão em Economia da Saúde.}

Antonia Eliane de Araújo
Enfermeira. Coordenadora da Atenção à Saúde da
Secretaria da Saúde de Reriutaba, Ceará. Especialista
em Psicopedagogia.

Lucineide Paulo de Carvalho

Enfermeira. Secretaria da Saúde de Reriutaba, Ceará. Especialista em Psicopedagogia e Saúde da Famíliat.

Vitória Ferreira do Amaral
Acadêmica de Enfermagem da Universidade Estadual
Vale do Acaraú (UVA).

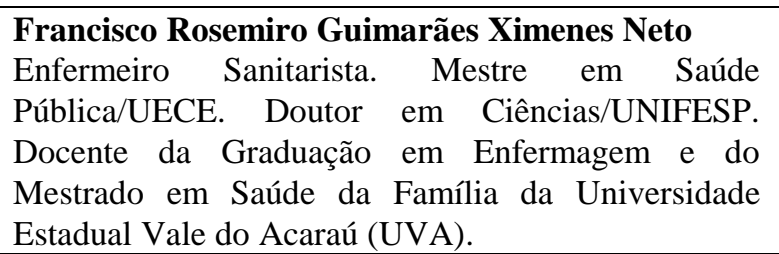

